PROCEEDINGS OF THE

AMERICAN MATHEMATICAL SOCIETY

Volume 130, Number 11, Pages 3393-3400

S 0002-9939(02)06538-3

Article electronically published on March 29, 2002

\title{
ASYMPTOTIC DIRICHLET PROBLEM FOR THE $p$-LAPLACIAN ON CARTAN-HADAMARD MANIFOLDS
}

\author{
ILKKA HOLOPAINEN
}

(Communicated by Juha M. Heinonen)

\begin{abstract}
We show the existence of nonconstant bounded $p$-harmonic functions on Cartan-Hadamard manifolds of pinched negative curvature by solving the asymptotic Dirichlet problem at infinity for the $p$-Laplacian. More precisely, we prove that given a continuous function $h$ on the sphere at infinity there exists a unique $p$-harmonic function $u$ on $M$ with boundary values $h$.
\end{abstract}

\section{INTRODUCTION}

In this paper we show the existence of nonconstant bounded $p$-harmonic functions on Cartan-Hadamard manifolds $M$ of pinched negative curvature by solving the asymptotic Dirichlet problem at infinity for the $p$-Laplacian. More precisely, we prove that given a continuous function $h$ on the sphere at infinity there exists a unique $p$-harmonic function $u$ on $M$ with boundary values $h$.

Let $M$ be a Cartan-Hadamard manifold, that is, a connected, simply connected, complete Riemannian $n$-manifold, $n \geq 2$, of nonpositive sectional curvature. By the Cartan-Hadamard theorem, the exponential map $\exp _{o}: T_{o} M \rightarrow M$ is a diffeomorphism for every point $o \in M$. In particular, $M$ is diffeomorphic to $\mathbb{R}^{n}$. It is well-known that $M$ can be compactified by adding a sphere at infinity, denoted by $S(\infty)$, so that the resulting space $\bar{M}=M \cup S(\infty)$ will be homeomorphic to a closed Euclidean ball. The sphere at infinity is defined as the set of all equivalent classes of geodesic rays in $M$; two geodesic rays $\gamma_{1}$ and $\gamma_{2}$ are equivalent if there exists a finite constant $c$ such that $d\left(\gamma_{1}(t), \gamma_{2}(t)\right) \leq c$ for all $t \geq 0$. There is a natural topology, called the cone topology, on $\bar{M}=\bar{M} \cup S(\infty)$ defined as follows. For any point $o \in M$ and $v \in T_{o} M$, let

$$
C_{o}(v, \alpha)=\left\{x \in M \backslash\{o\}: \varangle\left(v, \dot{\gamma}^{x}(0)\right)<\alpha\right\}
$$

be the cone about $v$ of angle $\alpha>0$, where $\gamma^{x}$ is the unique geodesic from $o=\gamma^{x}(0)$ to $x$ and $\varangle\left(v, \dot{\gamma}^{x}(0)\right)$ is the angle between vectors $v$ and $\dot{\gamma}^{x}(0)$ in $T_{o} M$. Then geodesic balls $B(q, r), q \in M, r>0$, and truncated cones

$$
T_{o}(v, \alpha, s)=C_{o}(v, \alpha) \backslash \bar{B}(o, s),
$$

with $v \in T_{0} M, \alpha>0, s>0$, form a basis for the cone topology. Furthermore, the cone topology is independent of the choice of $o \in M$ and, equipped with this

Received by the editors June 14, 2001.

2000 Mathematics Subject Classification. Primary 58J60; Secondary 53C20, 31C12.

Key words and phrases. Cartan-Hadamard manifold, p-harmonic function.

The author was supported in part by the Academy of Finland, projects 6355 and 44333.

(C)2002 American Mathematical Society 
topology, $\bar{M}$ is homeomorphic to the closed unit ball $\bar{B}^{n} \subset \mathbb{R}^{n}$ and $S(\infty)$ to the sphere $S^{n-1}=\partial B^{n}$; see [7]. In particular, given $o \in M, S(\infty)$ may be canonically identified with the unit sphere $S^{n-1} \subset T_{o} M$.

It is natural to ask whether every continuous function on $S(\infty)$ has a unique harmonic extension to $M$. This so-called asymptotic Dirichlet problem was solved by Choi if the sectional curvature has a negative upper bound $K \leq-a^{2}<0$ and any two points of the sphere at infinity can be separated by convex neighborhoods; see [6]. Such appropriate convex sets were constructed by Anderson [3] for manifolds of pinched sectional curvature $-b^{2} \leq K \leq-a^{2}<0$. The Dirichlet problem was independently solved by Sullivan [13] under the same curvature assumptions by using probabilistic arguments. In [4], Anderson and Schoen presented a simple and direct proof. Ancona 11 was able to replace the lower curvature bound by a bounded geometry assumption that each ball up to a fixed radius is bi-Lipschitz equivalent to an open set in $\mathbb{R}^{n}$. He also considered a more general class of operators. On the other hand, Ancona [2] showed that the Dirichlet problem cannot be solved, in general, if there are neither curvature lower bounds nor the bounded geometry assumption; see also [5]. In the general case of the $p$-Laplacian, the corresponding problem has been open so far. Pansu 11] has shown the existence of nonconstant bounded $p$-harmonic functions with finite $p$-energy on Cartan-Hadamard manifolds of pinched curvature $-b^{2} \leq K \leq-a^{2}$ if $p>(n-1) b / a$.

\section{Asymptotic Dirichlet problem}

Let $G \subset M$ be an open set and $1<p<\infty$. Recall that a function $u \in W_{\text {loc }}^{1, p}(G)$ is a (weak) solution of the equation

$$
-\operatorname{div}\left(|\nabla u|^{p-2} \nabla u\right)=0
$$

in $G$ if

$$
\int_{G}\left\langle|\nabla u|^{p-2} \nabla u, \nabla \varphi\right\rangle=0
$$

for all $\varphi \in C_{0}^{\infty}(G)$. Above $W_{\text {loc }}^{1, p}(G)$ is the (local) Sobolev space of all functions $u \in L_{\mathrm{loc}}^{p}(G)$ whose distributional gradient $\nabla u$ belongs to $L_{\mathrm{loc}}^{p}(G)$. Continuous solutions of (2.1) are called p-harmonic. It is well-known that every solution of (2.1) has a continuous representative by the fundamental work of Serrin 12. We say that a function $u \in W_{\text {loc }}^{1, p}(G)$ is a $p$-supersolution in $G$ if

$$
-\operatorname{div}\left(|\nabla u|^{p-2} \nabla u\right) \geq 0
$$

weakly in $G$, that is,

$$
\int_{G}\left\langle|\nabla u|^{p-2} \nabla u, \nabla \varphi\right\rangle \geq 0
$$

for all nonnegative $\varphi \in C_{0}^{\infty}(G)$. Furthermore, we say that $u$ is a $p$-subsolution if $-u$ is a $p$-supersolution.

In this section we show that the direct approach to solve the Dirichlet problem taken by Anderson and Schoen in [4 also works in the nonlinear setting of $p$ harmonic functions.

Theorem 2.1. Let $M$ be a Cartan-Hadamard manifold whose sectional curvature $K$ satisfies

$$
-b^{2} \leq K \leq-a^{2}
$$


for some constants $b \geq a>0$. Let $h$ be a continuous function on $S(\infty)$. Then there exists a unique function $u \in C(\bar{M})$ which is p-harmonic in $M$ and $u=h$ on $S(\infty)$.

The proof requires some preliminaries. Let $h \in C(S(\infty))$ be given. Fix a point $o \in M$ and write $r(x)=d(x, o)$. We identify $S(\infty)$ with the unit sphere $S^{n-1} \subset$ $T_{o} M$. Therefore, we may consider $h$ as a continuous function on $S^{n-1}$. Assume that $h: S^{n-1} \rightarrow \mathbb{R}$ is Lipschitz. We extend $h$ radially to a continuous function $\tilde{h}$ on $M \backslash\{o\}$. More precisely, we define $\tilde{h}$ in polar coordinates about $o$ by

$$
\tilde{h}(r, \vartheta)=h(\vartheta)
$$

for every $r>0$ and $\vartheta \in S^{n-1}$. The Lipschitz continuity of $h$ and the curvature upper bound $K \leq-a^{2}$ imply that

$$
\operatorname{osc}(\tilde{h}, B(x, 3)):=\sup _{B(x, 3)} \tilde{h}-\inf _{B(x, 3)} \tilde{h} \leq c L e^{-\operatorname{ar}(x)},
$$

where $L$ is the Lipschitz constant of $h$; see 44. Next we define a smooth function $h$ on $M$ such that

$$
\lim _{x \rightarrow \xi} h(x)=h(\xi)
$$

for every $\xi \in S(\infty)$ and that first and second order derivatives of $h$ are effectively controlled. For this purpose, we fix a maximal 1-separated set $Q=\left\{q_{1}, q_{2}, \ldots\right\} \subset$ $M$, that is,

$$
d\left(q_{i}, q_{j}\right) \geq 1
$$

whenever $i \neq j$ and no more points can be added to $Q$ without breaking the condition (2.5). We may assume that $o \notin Q$. In particular, the balls $B\left(q_{i}, 1 / 2\right)$ are mutually disjoint and $M=\bigcup_{i} B\left(q_{i}, 1\right)$. For each $x \in M$, we write $Q_{x}=Q \cap B(x, 3)$. The curvature lower bound then implies that

$$
\operatorname{card} Q_{x} \leq c,
$$

where $c$ is independent of $x$; see e.g. [10. Then we define

$$
h(x)=\sum_{q_{i} \in Q} \tilde{h}\left(q_{i}\right) \varphi_{i}(x),
$$

where $\left\{\varphi_{i}\right\}$ is a partition of unity subordinate to $\left\{B\left(q_{i}, 3\right)\right\}$ defined as follows. First choose a $C^{\infty}$ function $f:[0, \infty[\rightarrow[0,1]$ such that $f|[0,1]=1, f|[2, \infty[=0$, and

$$
\max \left\{\left|f^{\prime}(t)\right|,\left|f^{\prime \prime}(t)\right|\right\} \leq c \mathcal{X}_{[1,2]}(t)
$$

for some constant $c$, where $\mathcal{X}_{[1,2]}$ is the characteristic function of the interval $[1,2]$. For $q_{i} \in Q$ and $x \in M$, let $\eta_{i}(x)=f\left(r_{i}(x)\right)$, where $r_{i}(x)=d\left(x, q_{i}\right)$. Finally we set

$$
\varphi_{i}(x)=\frac{\eta_{i}(x)}{\sum_{j} \eta_{j}(x)} .
$$

To estimate first and second order derivatives of $h$, we first observe that

$$
\nabla \eta_{i}(x)=f^{\prime}\left(r_{i}(x)\right) \nabla r_{i}(x)
$$

and

$$
\begin{aligned}
\Delta \eta_{i}(x) & =f^{\prime}\left(r_{i}(x)\right) \Delta r_{i}(x)+\left\langle\nabla f^{\prime}\left(r_{i}(x)\right), \nabla r_{i}(x)\right\rangle \\
& =f^{\prime}\left(r_{i}(x)\right) \Delta r_{i}(x)+f^{\prime \prime}\left(r_{i}(x)\right)
\end{aligned}
$$


since $\left\langle\nabla r_{i}(x), \nabla r_{i}(x)\right\rangle=\left|\nabla r_{i}(x)\right|^{2} \equiv 1$. Thus (2.8) and (2.10) imply that

$$
\left|\nabla \eta_{i}(x)\right| \leq c \mathcal{X}_{A\left(q_{i} ; 1,2\right)}(x),
$$

where $A(y ; s, t)=\bar{B}(y, t) \backslash B(y, s)$. By the Hessian comparison theorem (8, Theorem A]),

$$
(n-1) a \operatorname{coth}\left(a r_{i}(x)\right) \leq \Delta r_{i}(x) \leq(n-1) b \operatorname{coth}\left(b r_{i}(x)\right) .
$$

Combining this with (2.8) and (2.11) yields

$$
\left|\Delta \eta_{i}(x)\right| \leq c \mathcal{X}_{A\left(q_{i} ; 1,2\right)}(x) .
$$

Since $\sum_{j} \eta_{j}(x) \geq 1,0 \leq \eta_{i}(x) \leq 1$, and $\operatorname{card} Q \cap B(x, 3) \leq c$ for every $x \in M$, we get by a simple computation that

$$
\left|\nabla \varphi_{i}(x)\right| \leq c \mathcal{X}_{B\left(q_{i}, 4\right)}(x)
$$

and

$$
\left|\Delta \varphi_{i}(x)\right| \leq c \mathcal{X}_{B\left(q_{i}, 4\right)}(x) .
$$

In the next lemma we collect those properties of $h$ that are crucial in the sequel.

Lemma 2.2. Let $r: M \rightarrow \mathbb{R}$ be the distance function $r(x)=d(x, o)$ and let $h: M \rightarrow \mathbb{R}$ be the function given by (2.7). Furthermore, let $v: M \backslash\{o\} \rightarrow \mathbb{R}$ be defined by

$$
v(x)=e^{-\delta r(x)},
$$

with $\delta>0$. Then there exists a constant $c_{0}$ independent of $h$ and $\delta$ such that

$$
\begin{gathered}
|\nabla h(x)| \leq c_{0} L e^{-a r(x)}, \\
|\Delta h(x)| \leq c_{0} L e^{-a r(x)}, \\
|\nabla\langle\nabla h, \nabla h\rangle(x)| \leq\left(c_{0} L\right)^{2} e^{-2 a r(x)}, \\
|\nabla\langle\nabla h, \nabla v\rangle(x)| \leq c_{0} L(1+\delta) \delta e^{-(a+\delta) r(x)}
\end{gathered}
$$

for $r(x) \geq 1$. Moreover,

$$
\lim _{x \rightarrow \xi} h(x)=h(\xi)
$$

for every $\xi \in S(\infty)$.

Proof. Fix $x \in M \backslash B(o, 1)$ and choose $q \in Q$ such that $x \in B(q, 1)$. Then

$$
\begin{aligned}
\nabla h(x) & =\sum_{q_{i} \in Q} \tilde{h}\left(q_{i}\right) \nabla \varphi_{i}(x)=\sum_{q_{i} \in Q_{x}} \tilde{h}\left(q_{i}\right) \nabla \varphi_{i}(x) \\
& =\sum_{q_{i} \in Q_{x}}\left(\tilde{h}\left(q_{i}\right)-\tilde{h}(q)\right) \nabla \varphi_{i}(x)
\end{aligned}
$$

since $\sum_{q_{i} \in Q_{x}} \varphi_{i}=1$ in a neighborhood of $x$ and therefore

$$
\sum_{q_{i} \in Q_{x}} \tilde{h}(q) \nabla \varphi_{i}(x)=\tilde{h}(q) \nabla\left(\sum_{q_{i} \in Q_{x}} \varphi_{i}\right)(x)=0 .
$$

By (2.4), (2.6), and (2.12),

$$
|\nabla h(x)| \leq c\left(\operatorname{card} Q_{x}\right) \operatorname{osc}(\tilde{h}, B(x, 3)) \leq c L e^{-a r(x)}
$$


which proves (2.15). By a similar argument using (2.13) instead of (2.12) we obtain (2.16). For the proof of the estimate (2.17), we first observe that

$$
\begin{aligned}
\langle\nabla h, \nabla h\rangle(x) & =\left\langle\sum_{q_{i} \in Q_{x}}\left(\tilde{h}\left(q_{i}\right)-\tilde{h}(q)\right) \nabla \varphi_{i}, \sum_{q_{j} \in Q_{x}}\left(\tilde{h}\left(q_{j}\right)-\tilde{h}(q)\right) \nabla \varphi_{j}\right\rangle(x) \\
& =\sum_{q_{i}, q_{j} \in Q_{x}}\left(\tilde{h}\left(q_{i}\right)-\tilde{h}(q)\right)\left(\tilde{h}\left(q_{j}\right)-\tilde{h}(q)\right)\left\langle\nabla \varphi_{i}, \nabla \varphi_{j}\right\rangle(x),
\end{aligned}
$$

and so

$$
\nabla\langle\nabla h, \nabla h\rangle(x)=\sum_{q_{i}, q_{j} \in Q_{x}}\left(\tilde{h}\left(q_{i}\right)-\tilde{h}(q)\right)\left(\tilde{h}\left(q_{j}\right)-\tilde{h}(q)\right) \nabla\left\langle\nabla \varphi_{i}, \nabla \varphi_{j}\right\rangle(x) .
$$

By (2.4) and (2.6) it suffices to prove that

$$
\left|\nabla\left\langle\nabla \varphi_{i}, \nabla \varphi_{j}\right\rangle(x)\right| \leq c
$$

for all $q_{i}, q_{j} \in Q_{x}$ which reduces to establishing that

$$
\left|\nabla\left\langle\nabla r_{i}, \nabla r_{j}\right\rangle(x)\right| \leq c
$$

whenever $x \in A\left(q_{i} ; 1,2\right) \cap A\left(q_{j} ; 1,2\right)$. Let $X_{1}, \ldots, X_{n}$ be an orthonormal frame in a neighborhood of $x$. Then

$$
\begin{aligned}
\nabla\left\langle\nabla r_{i}, \nabla r_{j}\right\rangle & =\sum_{k}\left(X_{k}\left\langle\nabla r_{i}, \nabla r_{j}\right\rangle\right) X_{k} \\
& =\sum_{k}\left(\left\langle\nabla_{X_{k}} \nabla r_{i}, \nabla r_{j}\right\rangle+\left\langle\nabla r_{i}, \nabla_{X_{k}} \nabla r_{j}\right\rangle\right) X_{k} .
\end{aligned}
$$

On the other hand,

$$
\left\langle\nabla_{X_{k}} \nabla r_{i}, \nabla r_{j}\right\rangle=\nabla^{2} r_{i}\left(X_{k}, \nabla r_{j}\right)
$$

where $\nabla^{2} r_{i}$ is the Hessian of $r_{i}$. By the Hessian comparison theorem all eigenvalues of $\nabla^{2} r_{i}$ are nonnegative and bounded from above by $b \operatorname{coth}\left(b r_{i}\right)$. Hence

$$
\left|\left\langle\nabla_{X_{k}} \nabla r_{i}, \nabla r_{j}\right\rangle(x)\right| \leq b \operatorname{coth}\left(b r_{i}(x)\right)\left|X_{k}(x)\right|\left|\nabla r_{j}(x)\right|=b \operatorname{coth}\left(b r_{i}(x)\right) \leq c
$$

if $r_{i}(x) \geq 1$. Similarly, $\left|\left\langle\nabla_{X_{k}} \nabla r_{j}, \nabla r_{i}\right\rangle(x)\right| \leq c$ if $r_{j}(x) \geq 1$, and so (2.20) follows. This proves (2.17). The estimate (2.18) can be established similarly since

$$
\begin{aligned}
|\nabla\langle\nabla h, \nabla v\rangle(x)| & \leq \delta e^{-\delta r(x)} \sum_{q_{i} \in Q_{x}}\left|\tilde{h}\left(q_{i}\right)-\tilde{h}(q)\right|\left|\nabla\left\langle\nabla \varphi_{i}, \nabla r\right\rangle(x)\right| \\
& +\delta^{2} e^{-\delta r(x)}|\nabla r(x)| \sum_{q_{i} \in Q_{x}}\left|\tilde{h}\left(q_{i}\right)-\tilde{h}(q)\right|\left|\left\langle\nabla \varphi_{i}, \nabla r\right\rangle(x)\right| .
\end{aligned}
$$

Now $\left|\nabla\left\langle\nabla \varphi_{i}, \nabla r\right\rangle(x)\right| \leq c$ if $r(x) \geq 1$ by a similar argument as above, and thus (2.18) follows. Finally, (2.19) follows easily from the definition (2.7) and from the continuity of $h \mid S(\infty)$.

Lemma 2.3. Suppose that $h: S^{n-1} \rightarrow \mathbb{R}$ is L-Lipschitz, where $S^{n-1}$ is the unit sphere in $T_{o} M$. Define $h: M \rightarrow \mathbb{R}$ by (2.7) and let $v=e^{-\delta r}$. Then there exist $\left.\delta_{0} \in\right] 0, a[$ such that, for every $\left.\delta \in] 0, \delta_{0}\right], h+v$ is a p-supersolution and $h-v$ is a p-subsolution in $M \backslash \bar{B}\left(o, R_{\delta}\right)$, where $R_{\delta}=R_{\delta}\left(a, \delta, c_{0}, L\right)$. 
Proof. In what follows $R_{1}, \ldots, R_{5}$ are constants depending only on $a, \delta, c_{0}$, and $L$. Since $h$ and $v$ are smooth in $M \backslash\{o\}$, we can prove the claims by direct computation using the properties of $h$ and $v$ given by Lemma 2.2. Write $u=h+v$ and note that $\nabla u=\nabla h-\delta e^{-\delta r} \nabla r \neq 0$ if $\delta<a$ and $r>R_{1}$ by (2.15). Hence

$$
\operatorname{div}\left(|\nabla u|^{p-2} \nabla u\right)=|\nabla u|^{p-2} \Delta u+\frac{p-2}{2}|\nabla u|^{p-4}\left\langle\nabla\left(|\nabla u|^{2}\right), \nabla u\right\rangle
$$

in $M \backslash \bar{B}\left(o, R_{1}\right)$. Next we deduce from 2.11) that

$$
\Delta v=-\delta e^{-\delta r} \Delta r+\delta^{2} e^{-\delta r} \leq \delta e^{-\delta r}(\delta-(n-1) a) \leq-c_{1} \delta e^{-\delta r}<0,
$$

with $c_{1}=(n-1) a / 2$ whenever $\delta \leq(n-1) a / 2$; cf. [4]. Given $\delta<a$ there exists $R_{2}$ such that

$$
\begin{aligned}
\delta^{2} e^{-2 \delta r} \leq|\nabla h+\nabla v|^{2} & =|\nabla h|^{2}+2\langle\nabla h, \nabla v\rangle+|\nabla v|^{2} \\
& \leq\left(c_{0} L\right)^{2} e^{-2 a r}+2 c_{0} L \delta e^{-(a+\delta) r}+\delta^{2} e^{-2 \delta r} \\
& \leq 2 \delta^{2} e^{-2 \delta r}
\end{aligned}
$$

as soon as $r \geq R_{2}$. Hence

$$
d_{p}^{-1} \delta^{p-2} e^{-\delta(p-2) r} \leq|\nabla h+\nabla v|^{p-2} \leq d_{p} \delta^{p-2} e^{-\delta(p-2) r},
$$

where $d_{p}=2^{|p-2| / 2}$. If $\delta<a \wedge c_{1}$, we get an estimate

$$
|\nabla h+\nabla v|^{p-2}(\Delta h+\Delta v) \leq d_{p}^{-1} \delta^{p-2} e^{-\delta(p-2) r}\left[d_{p}^{2} c_{0} L e^{-a r}-c_{1} \delta e^{-\delta r}\right]
$$

for the first term in the right-hand side of (2.21). To estimate the second term in (2.21) we write

$$
\begin{aligned}
\left\langle\nabla\left(|\nabla u|^{2}\right), \nabla u\right\rangle & =\left\langle\nabla\left(|\nabla h|^{2}\right), \nabla u\right\rangle+\left\langle\nabla\left(|\nabla v|^{2}\right), \nabla u\right\rangle+2\langle\nabla\langle\nabla h, \nabla v\rangle, \nabla u\rangle \\
& =A+B+C .
\end{aligned}
$$

By (2.15) and (2.17),

$$
\begin{aligned}
A & =\left\langle\nabla\left(|\nabla h|^{2}\right), \nabla h+\nabla v\right\rangle \\
& \leq|\nabla\langle\nabla h, \nabla h\rangle||\nabla h+\nabla v| \\
& \leq\left(c_{0} L\right)^{2} e^{-2 a r}\left(c_{0} L e^{-a r}+\delta e^{-\delta r}\right) \\
& \leq \delta^{4} e^{-3 \delta r}
\end{aligned}
$$

if $r \geq R_{3}$. Similarly,

$$
\begin{aligned}
B & =\left\langle\nabla\left(|\nabla v|^{2}\right), \nabla h+\nabla v\right\rangle \\
& \leq 2 c_{0} L \delta^{3} e^{-2 \delta r} e^{-a r}+2 \delta^{4} e^{-3 \delta r} \\
& \leq 3 \delta^{4} e^{-3 \delta r}
\end{aligned}
$$

if $r \geq R_{4}$. Finally, (2.18) and (2.15) imply that

$$
\begin{aligned}
C & =2\langle\nabla(\langle\nabla h, \nabla v\rangle), \nabla h+\nabla v\rangle \\
& \leq 2|\nabla\langle\nabla h, \nabla v\rangle \| \nabla h+\nabla v| \\
& \leq 2 c_{0} L(1+\delta) \delta e^{-(a+\delta) r}\left(c_{0} L e^{-a r}+\delta e^{-\delta r}\right) \\
& \leq \delta^{4} e^{-3 \delta r}
\end{aligned}
$$


whenever $r \geq R_{5}$. Putting these estimates together yields

$$
\begin{aligned}
\operatorname{div}\left(|\nabla u|^{p-2} \nabla u\right) & =|\nabla u|^{p-2} \Delta u+\frac{p-2}{2}|\nabla u|^{p-4}\left\langle\nabla\left(|\nabla u|^{2}\right), \nabla u\right\rangle \\
& \leq d_{p}^{-1} \delta^{p-2} e^{-\delta(p-2) r}\left[d_{p}^{2} c_{0} L e^{-a r}-\left(c_{1}-\delta C_{p}\right) \delta e^{-\delta r}\right],
\end{aligned}
$$

where

$$
C_{p}=3|p-2| 2^{\frac{|p-2|+|p-4|}{2}} .
$$

Choosing $\delta_{0}<\min \left\{a, c_{1}, c_{1} /\left(2 C_{p}\right)\right\}$, with an obvious interpretation $c_{1} /\left(2 C_{p}\right)=\infty$ if $p=2$, finally gives an estimate

$$
\operatorname{div}\left(|\nabla u|^{p-2} \nabla u\right) \leq-c_{2} \delta^{p-1} e^{-\delta(p-1) r}<0
$$

if $\delta \leq \delta_{0}$ and $r \geq R_{\delta}$. Similarly, we obtain an estimate

$$
\operatorname{div}\left(|\nabla h-\nabla v|^{p-2}(\nabla h-\nabla v)\right) \geq c_{2} \delta^{p-1} e^{-\delta(p-1) r}>0
$$

if $\delta \leq \delta_{0}$ and $r \geq R_{\delta}$.

Lemma 2.4. Identify $S(\infty)$ with the unit sphere $S^{n-1} \subset T_{o} M$. Assume that $h$ : $S^{n-1} \rightarrow \mathbb{R}$ is L-Lipschitz. Then there exists a p-harmonic function $u$ in $M$ satisfying

$$
\lim _{x \rightarrow \xi} u(x)=h(\xi)
$$

for every $\xi \in S(\infty)$.

Proof. Define $h: M \rightarrow \mathbb{R}$ by (2.7) and let $\left.\delta \in] 0, \delta_{0}\right]$ and $R_{\delta}$ be given by Lemma 2.3 First we note that $h$ is bounded, and therefore we can choose a constant $\lambda \in] 0,1]$ such that

$$
\lambda \operatorname{osc}(h, M) \leq e^{-\delta R_{\delta}} .
$$

Since $\lambda h \mid S^{n-1}$ is also $L$-Lipschitz, $\lambda h+v$ is a $p$-supersolution and $\lambda h-v$ is a $p$-subsolution in $M \backslash \bar{B}\left(o, R_{\delta}\right)$. For $i=1,2, \ldots$, let $u_{i} \in C(M)$ be the unique function such that $u_{i}$ is $p$-harmonic in $B\left(o, 2^{i} R_{\delta}\right)$ and $u_{i} \equiv \lambda h$ in $M \backslash B\left(o, 2^{i} R_{\delta}\right)$. Now $\lambda h-v \leq u_{i} \leq \lambda h+v$ on $\partial\left(B\left(o, 2^{i} R_{\delta}\right) \backslash \bar{B}\left(o, R_{\delta}\right)\right)$, and hence the same holds in $B\left(o, 2^{i} R_{\delta}\right) \backslash \bar{B}\left(o, R_{\delta}\right)$ by the comparison principle; see [9, 3.18 and 7.6]. Hence there exists a subsequence, denoted again by $\left(u_{i}\right)$ and a function $u \in C(M)$ such that $\lambda^{-1} u_{i} \rightarrow u$ locally uniformly in $M$. Furthermore, the function $u$ is $p$-harmonic in $M$ and satisfies (2.22) for every $\xi \in S(\infty)$.

Proof of Theorem 2.1. Fix $o \in M$ and identify $S(\infty)$ with $S^{n-1} \subset T_{o} M$. Let $\left(h_{i}\right)$ be a sequence of Lipschitz functions on $S^{n-1}$ such that $h_{i} \rightarrow h$ uniformly on $S^{n-1}$. By Lemma 2.4 there are $p$-harmonic functions $u_{i} \in C(\bar{M})$ with $u_{i}=h_{i}$ in $S(\infty)$. The sequence $\left(u_{i}\right)$ converges uniformly in $\bar{M}$ to a function $u \in C(\bar{M})$ which is $p$-harmonic in $M$ and $u=h$ in $S(\infty)$. To prove the uniqueness, suppose that $u$ and $w$ are both $p$-harmonic in $M$, continuous in $\bar{M}$, with $u=w$ in $S(\infty)$, and $u(y)>w(y)$ for some $y \in M$. Let $\varepsilon=(u(y)-w(y)) / 2$. Since $u$ and $w$ are continuous in $\bar{M}$ and they coincide on the compact set $S(\infty)$, there exists $R>0$ such that $|u(x)-w(x)|<\varepsilon$ for every $x \in M \backslash B(o, R)$. Let $D$ be the $y$-component of $\{x \in M: u(x)>w(x)+\varepsilon\}$. It follows that $D$ is a relatively compact domain in $M$ and $u=w+\varepsilon$ on $\partial D$. Hence $u=w+\varepsilon$ in $D$ which leads to a contradiction since $y \in D$. This proves the uniqueness and thus the whole theorem is proved. 


\section{REFERENCES}

[1] Ancona, A. Negatively curved manifolds, elliptic operators, and the Martin boundary. Ann. of Math. (2) 125, 3 (1987), 495-536. MR 88k:58160

[2] Ancona, A. Convexity at infinity and Brownian motion on manifolds with unbounded negative curvature. Rev. Mat. Iberoamericana 10, 1 (1994), 189-220. MR 95a:58132

[3] Anderson, M. T. The Dirichlet problem at infinity for manifolds of negative curvature. J. Differential Geom. 18, 4 (1983), 701-721 (1984). MR 85m:58178

[4] Anderson, M. T., And Schoen, R. Positive harmonic functions on complete manifolds of negative curvature. Ann. of Math. (2) 121, 3 (1985), 429-461. MR 87a:58151

[5] Borbély, A. The nonsolvability of the Dirichlet problem on negatively curved manifolds. Differential Geom. Appl. 8, 3 (1998), 217-237. MR 99j:53043

[6] CHOI, H. I. Asymptotic Dirichlet problems for harmonic functions on Riemannian manifolds. Trans. Amer. Math. Soc. 281, 2 (1984), 691-716. MR 85b:53040

[7] Eberlein, P., and O'Neill, B. Visibility manifolds. Pacific J. Math. 46 (1973), 45-109. MR 49:1421

[8] Greene, R. E., And Wu, H. Function theory on manifolds which possess a pole. Springer, Berlin, 1979. MR 81a:53002

[9] Heinonen, J., Kilpeläinen, T., And Martio, O. Nonlinear potential theory of degenerate elliptic equations. The Clarendon Press Oxford University Press, New York, 1993. Oxford Science Publications. MR 94e:31003

[10] KANAI, M. Rough isometries, and combinatorial approximations of geometries of noncompact Riemannian manifolds. J. Math. Soc. Japan 37, 3 (1985), 391-413. MR 87d:53082

[11] Pansu, P. Cohomologie $L^{p}$ des variétés à courbure négative, cas du degré 1. Rend. Sem. Mat. Univ. Politec. Torino, Special Issue (1989), 95-120 (1990). Conference on Partial Differential Equations and Geometry (Torino, 1988). MR 92e:58200

[12] SERRIN, J. Local behavior of solutions of quasi-linear equations. Acta Math. 111 (1964), 247-302. MR 30:337

[13] Sullivan, D. The Dirichlet problem at infinity for a negatively curved manifold. J. Differential Geom. 18, 4 (1983), 723-732 (1984). MR 85m:58177

Department of Mathematics, P.O. Box 4 (Yliopistonkatu 5), Fin-00014 University of HeLsinki, Finland

E-mail address: ilkka.holopainen@helsinki.fi 